\title{
Magnetic properties of copper metaborate $\mathrm{CuB}_{2} \mathrm{O}_{4}$
}

\author{
G. A. Petrakovskiǐ, A. I. Pankrats,* M. A. Popov, A. D. Balaev, D. A. Velikanov, \\ A. M. Vorotynov, and K. A. Sablina
}

L. V. Kirenskiu Institute of Physics, Siberian Branch of the Russian Academy of Sciences, Akademgorodok, 660036 Krasnoyarsk, Russia

B. Roessli, J. Schefer, A. Amato, and U. Staub

Laboratory for Neutron Scattering, ETH Zurich \& Paul Scherrer Institute, CH-6232 Villigen PSI, Switzerland

M. Boehm

Laboratory for Neutron Scattering, ETH Zurich \& Paul Scherrer Institute, CH-6232 Villigen PSI, Switzerland; Institut Laue-Langevin, 38042 Grenoble, Cedex 9, France

B. Ouladdiaf

Institut Laue-Langevin, 38042 Grenoble, Cedex 9, France

(Submitted February 4, 2002)

Fiz. Nizk. Temp. 28, 840-849 (August-September 2002)

The results of experimental research on the magnetic and resonance properties, heat capacity, muon spin relaxation, and neutron scattering of single-crystal copper metaborate $\mathrm{CuB}_{2} \mathrm{O}_{4}$ are reviewed. The results of a symmetry analysis and of modeling by the method of the phenomenological thermodynamic potential are presented. The magnetic structure of the crystal in various temperature intervals of the magnetic ordering region is discussed. (C) 2002 American Institute of Physics. [DOI: 10.1063/1.1511704]

\section{INTRODUCTION}

The discovery of high-temperature superconductivity has stimulated a heightened interest in research on the magnetic properties of copper oxide compounds that, though not superconducting, have fragments of crystal structure identical to those of high- $T_{c}$ superconductors (HTSCs). A peculiarity of the divalent copper ion is that it forms a greater diversity of magnetic structures than any other chemical element. As an example of the wide range of magnetic properties of copper oxide compounds we can cite the oxocuprates which we investigated previously, among which one encounters a three-dimensional antiferromagnet with a four-spin exchange interaction $\left(\mathrm{Bi}_{2} \mathrm{CuO}_{4}\right),{ }^{1}$ a spin-Peierls chain magnet $\left(\mathrm{CuGeO}_{3}\right),{ }^{2,3}$ a two-dimensional antiferromagnet with a broken ladder structure $\left(\mathrm{LiCu}_{2} \mathrm{O}_{2}\right),{ }^{4}$ and a spin glass $\left(\mathrm{CuGa}_{2} \mathrm{O}_{4}\right){ }^{5}$ Research has begun on the triclinic magnet $\mathrm{Cu}_{5} \mathrm{Bi}_{2} \mathrm{~B}_{4} \mathrm{O}_{14}$, which has a ferro- or ferrimagnetic structure. ${ }^{6}$

We have recently grown single crystals of copper metaborate $\mathrm{CuB}_{2} \mathrm{O}_{4}$. The first studies of the physical properties of this compound show that in the temperature region below $20 \mathrm{~K}$ it has a complex state diagram, in certain regions of which the compound has a modulated (incommensurate) magnetic structure. In the majority of cases such structures arise as a result of a competition of exchange excitations. ${ }^{7}$ In these cases the crystal structure does not impose any restrictions on the possibility of realization of such magnetic structures. Dzyaloshinskiu first pointed out the possibility for modulated structures to arise on account of relativistic interactions. ${ }^{8}$ The physical cause of the onset of incommensurate structures in these cases is an antisymmetric
Dzyaloshinskiı̆-Moriya exchange interaction. Formally such magnetic structures can be described by including in the thermodynamic potential a Lifshitz invariant ${ }^{7}$ containing linearly the first derivatives of the two-component order parameter with respect to the coordinates. It should be noted that in this case an important restriction is placed on the symmetry of the crystal: the Lifshitz invariant can be included in the thermodynamic potential only for crystals lacking a center of inversion.

The incommensurate structure arising in copper metaborate belongs to this less-common type of modulated structure due to relativistic interactions. It is therefore of great interest to do comprehensive studies of such structures on highquality single crystals.

In this paper we present the results of experimental studies of the magnetic and certain other physical properties of copper metaborate $\mathrm{CuB}_{2} \mathrm{O}_{4}$ and discuss the magnetic structure of the crystal in different temperature intervals of the magnetic ordering region.

\section{EXPERIMENTAL DATA}

The technology for growing large, high-quality single crystals of copper metaborate is set forth in Ref. 9. X-ray and neutron diffraction studies ${ }^{10}$ at room temperature have shown that $\mathrm{CuB}_{2} \mathrm{O}_{4}$ is a tetragonal crystal, space group $I \overline{4} 2 d$, with lattice parameters $a=11.528 \AA$ and $c=5.607 \AA$. The unit cell contains 12 formula units (Fig. 1). The copper ions $\mathrm{Cu}^{2+}$ occupy two nonequivalent positions: $\mathrm{Cu}(b)$-the $4 b$ position, point symmetry $S_{4}(0,0,1 / 2)$, and $\mathrm{Cu}(d)$ - the $8 d$ position, point symmetry $C_{2}(0.0815,1 / 4,1 / 8)$. The $\mathrm{Cu}(b)$ ion is 


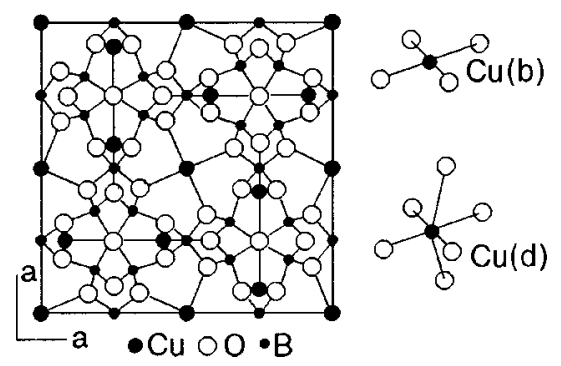

FIG. 1. Crystal structure of copper metaborate.

located at the center of a square formed by four oxygen ions; the $\mathrm{Cu}(d)$ ion is surrounded by six oxygen ions localized at the vertices of a distorted octahedron. A special highresolution neutron diffraction study ${ }^{10}$ showed that the crystal does not undergo any structure phase transitions down to a temperature of $1.5 \mathrm{~K}$.

Magnetic measurements made on the crystals showed that the magnetic susceptibility has sharp features at the temperatures $T_{I}=10 \mathrm{~K}$ and $T_{N}=20 \mathrm{~K}$. The results of a magnetic susceptibility measurement ${ }^{11}$ on a SQUID magnetometer for orientations of the magnetic field along and perpendicular to the tetragonal axis of the crystal are shown in Fig. 2. One notices the sharp anisotropy of the susceptibility. For a magnetic field applied in the basal plane of the crystal a jump in the susceptibility is observed at a temperature of $20 \mathrm{~K}$, and the susceptibility grows rapidly as the temperature is decreased further. At the temperature $T_{I}$ the susceptibility decreases abruptly by approximately an order of magnitude and then increases monotonically on further decrease of the temperature to $4.2 \mathrm{~K}$. For a magnetic field applied along the tetragonal axis of the crystal the temperature dependence of the susceptibility is smooth throughout the whole temperature range. The paramagnetic Curie temperature and the effective magnetic moment of the copper ion, determined from the high-temperature part of the magnetic susceptibility, have the values $\theta=-9.5 \mathrm{~K}$ and $\mu_{\text {eff }}=1.77 \mu_{B}$, respectively.

The field dependence of the magnetization measured on a vibrating magnetometer ${ }^{12}$ with a magnetic field up to 60 kOe oriented along the tetragonal axis of the crystal is smooth throughout the investigated temperature interval. At the same time, for a field orientation in the tetragonal plane

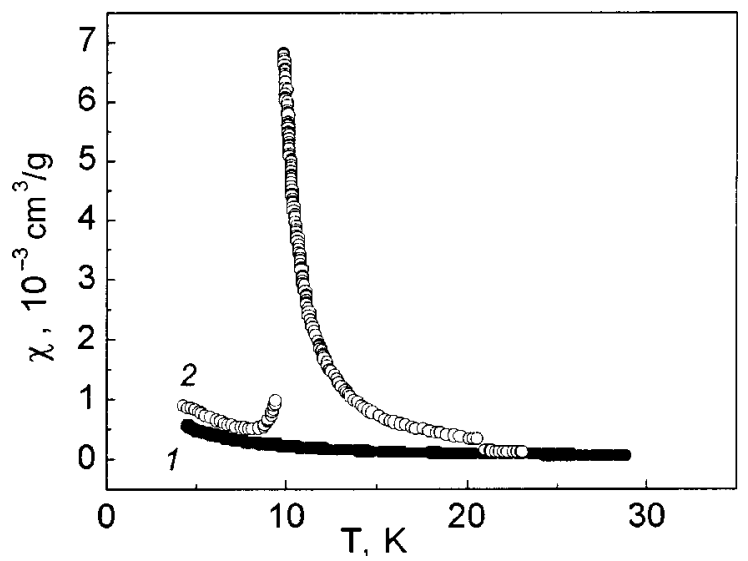

FIG. 2. Temperature dependence of the magnetic susceptibility of a copper metaborate single crystal. The magnetic field was parallel to the tetragonal axis of the crystal (1) and to the basal plane of the crystal (2).

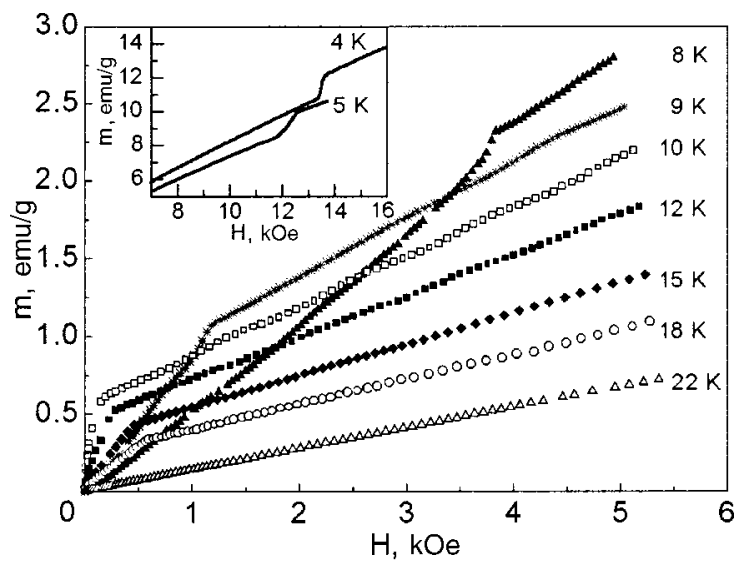

FIG. 3. Field dependence of the magnetization for $\mathbf{H} \perp \mathbf{c}$.

there are kinks on the magnetization curves (Fig. 3). In the temperature region $10-18 \mathrm{~K}$ the magnetization curves attest to the existence of a spontaneous magnetic moment in the basal plane of the crystal. The value of the spontaneous moment is $m_{0}=0.56 \mathrm{emu} / \mathrm{g}$ at $T=10 \mathrm{~K}$. When the temperature is lowered below $T_{I}$ the spontaneous magnetization vanishes, and a feature appears on the magnetization curves at higher magnetic fields. The linear approximation of the high-field linear parts of the field dependence of the magnetization in the temperature interval $4.2-10 \mathrm{~K}$ shows that above these fields a spontaneous magnetic moment again appears in the crystal.

The anomalies of the magnetic susceptibility described above are accompanied by features of the temperature dependence of the heat capacity (Fig. 4). ${ }^{11}$ The temperature dependence of the heat capacity exhibits two anomalies at temperatures coinciding with the anomalies of the magnetic susceptibility. In addition, at a temperature below $4 \mathrm{~K}$ the heat capacity has a feature in the form a broad maximum.

The data of muon spin relaxation $(\mu \mathrm{SR})$ measurements in Ref. 11 also confirm the presence of magnetic transformations at temperatures of 20 and $10 \mathrm{~K}$. Later measurements ${ }^{13}$ down to temperatures $\sim 0.1 \mathrm{~K}$ reveal an additional magnetic transformation at a temperature below $2 \mathrm{~K}$ (Fig. 5). It can be

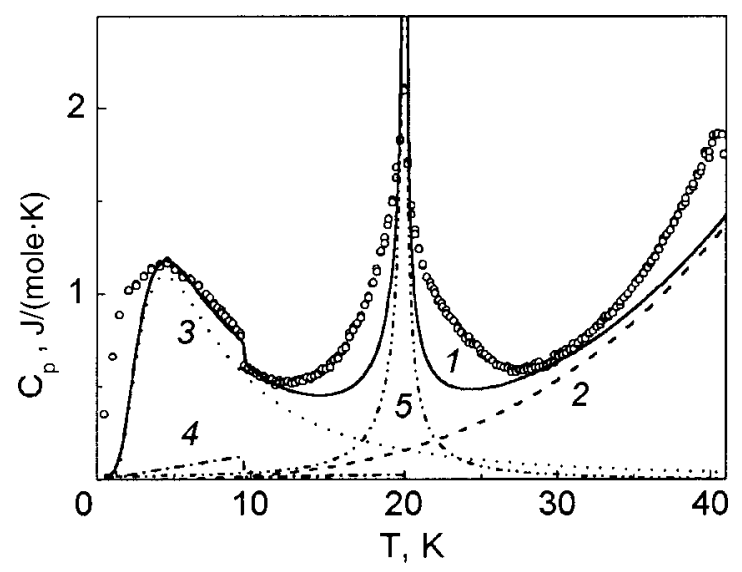

FIG. 4. Temperature dependence of the heat capacity of a single crystal of copper metaborate: the unfilled circles are experimental, ${ }^{11}$ and the curves are: 1-the result of the modeling, 2-the Debye contribution, 3-an anomaly of the Schottky type, 4-the Landau contribution, 5-the Ginzburg estimate. 


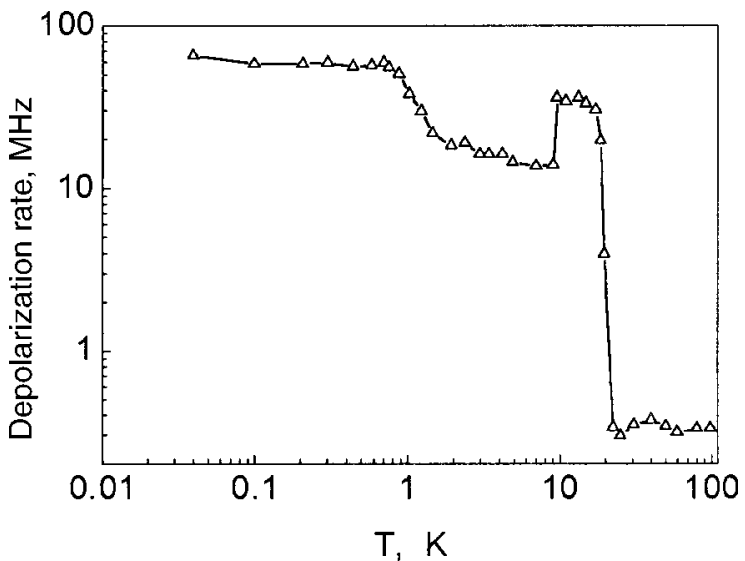

FIG. 5. Temperature dependence of the muon spin relaxation in copper metaborate.

assumed that this temperature also corresponds to a reorganization of the spin system of copper metaborate.

Magnetic resonance in $\mathrm{CuB}_{2} \mathrm{O}_{4}$ has been investigated in a wide range of frequencies, magnetic fields, and temperatures. ${ }^{14}$ Figure 6 shows the frequency-field curves of the magnetic resonance in $\mathrm{CuB}_{2} \mathrm{O}_{4}$ measured at $T=4.2 \mathrm{~K}$ for two orientations of the magnetic field: $\mathbf{H} \| \mathbf{c}$ and $\mathbf{H} \perp \mathbf{c}$. For $\mathbf{H} \| \mathbf{c}$ the dependence is smooth and almost linear. For the orientation $\mathbf{H} \perp \mathbf{c}$, however, a jump of the frequency is observed at $H_{c \perp}=12 \mathrm{kOe}$ as the field is increased (see inset "a"). This jump is due to a phase transition from the lowtemperature state 1 to a state 2 with a field-induced spontaneous magnetic moment at $H=H_{c \perp}$. Inset "b" shows the temperature dependence of the resonance field measured at different frequencies for $\mathbf{H} \perp \mathbf{c}$. For measurements at frequencies of 10.6 and $28.65 \mathrm{GHz}$ in the low-temperature region one observes sharp changes of the resonance field that are also consistent with a phase transition. The temperatures at which these changes occur decreases with increasing frequency. For the measurement at frequency $56.59 \mathrm{GHz}$ no anomalies of the resonance field exist down to $4.2 \mathrm{~K}$.

Figure 7 shows the $H_{\perp}-T$ phase diagram obtained from the data of the resonance and magnetic measurements. The

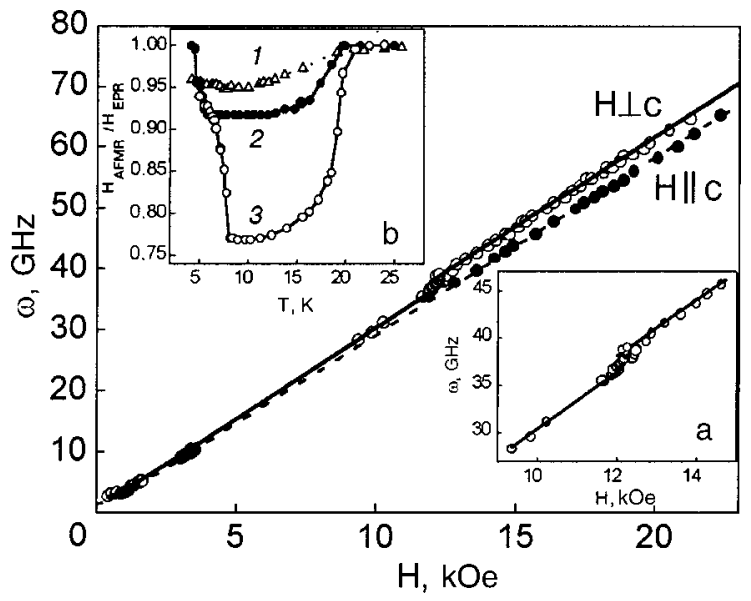

FIG. 6. Frequency-field curves of the magnetic resonance at $T=4.2 \mathrm{~K}$ for $\mathbf{H} \| \mathbf{c}$ and $\mathbf{H} \perp \mathbf{c}$. Inset "a" shows a fragment of the frequency-field curve for $\mathbf{H} \perp \mathbf{c}$. Inset "b" shows the temperature dependence of the resonance field for frequencies $\omega[\mathrm{GHz}]: 56.59$ (1), 28.65 (2), 10.6 (3).

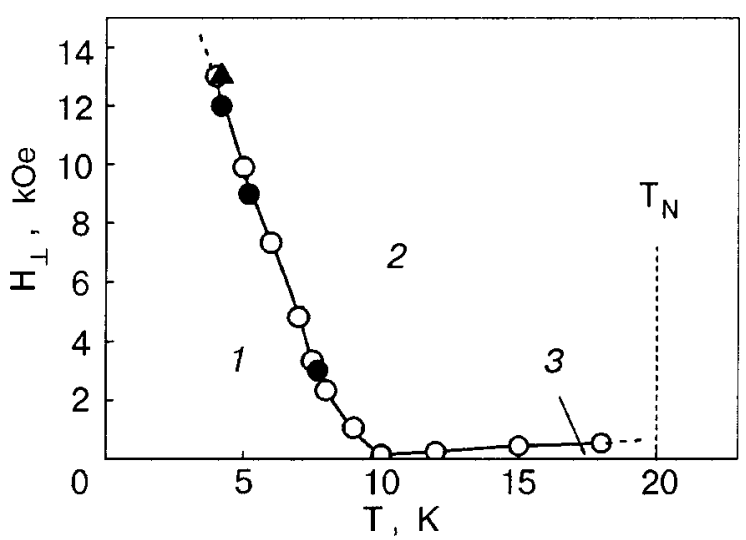

FIG. 7. $H_{\perp}-T$ phase diagram of the states of copper metaborate: magnetic $(\bigcirc)$ and resonance $(\boldsymbol{O})$ measurements; the data of Ref. $15(\boldsymbol{\Delta})$.

dashed line corresponds to a transition from the paramagnetic state to the magnetically ordered state 2 . It is clear from the phase diagram that the absence of low-temperature anomalies of the resonance parameters at frequency 56.59 $\mathrm{GHz}$ is due to the fact that at $T=4.2 \mathrm{~K}$ the $\mathrm{CuB}_{2} \mathrm{O}_{4}$ crystal is already found in state 2 , since the resonance field corresponding to this frequency, $H \approx 18 \mathrm{kOe}$, is higher than the critical value $H_{c \perp}=12 \mathrm{kOe}$. In addition, analysis of the resonance and magnetic data have shown ${ }^{14}$ that at the transition from state 2 to state 1 the magnetic moments of the copper ions remain in the basal plane, but the spontaneous magnetic moment vanishes. In this study we have made the assumption that a helicoidal (modulated) magnetic structure is realized in the low-temperature state 1 of $\mathrm{CuB}_{2} \mathrm{O}_{4}$. A helicoidal magnetic structure in the low-temperature state of $\mathrm{CuB}_{2} \mathrm{O}_{4}$ is evidenced by the absence of angular dependence of the resonance parameters in the basal plane in fields below $H_{c \perp}$ and the presence of angular dependence in fields above $H_{c \perp}$ (Fig. 8). For a simple helix in the absence of external magnetic field the local antiferromagnetic vectors are distributed uniformly over all directions in the basal plane. An external magnetic field deforms the helix, transforming it into a fan structure in which the antiferromagnet vectors are distributed within a sector with angular size $\alpha$. If the value of $\alpha$ is comparable to the period of the tetragonal angular dependence, $\pi / 2$, then averaging over all the local positions will

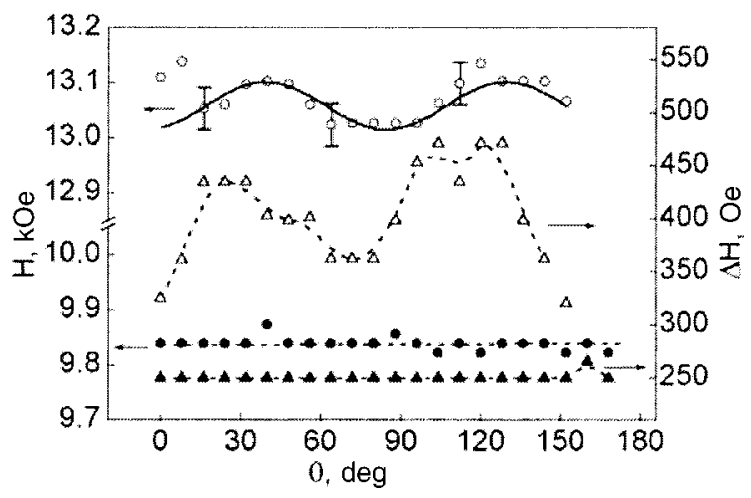

FIG. 8. Angular dependence of the resonance field and of the linewidth in the tetragonal plane at $T=4.2 \mathrm{~K}$ and frequencies [GHz]: $41.51(\bigcirc, \triangle)$; $29.008(\boldsymbol{\bullet}, \mathbf{\Delta})$. 


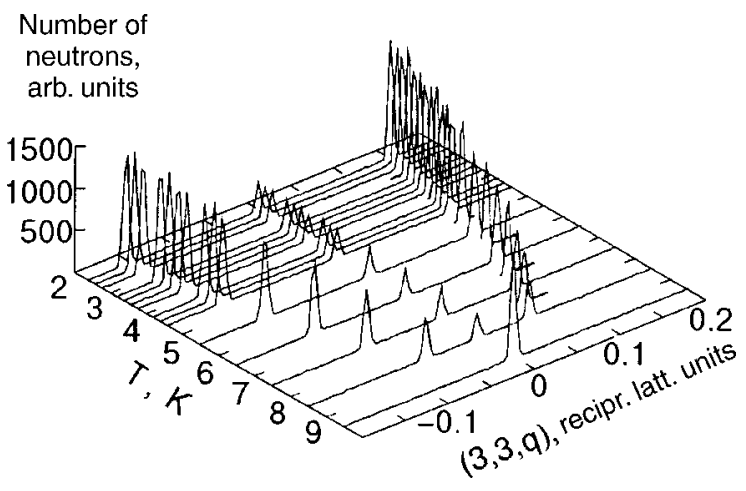

FIG. 9. Temperature dependence of the position of the magnetic satellites $(3,3,0+q)$ and $(3,3,0-q)$.

lead to the absence of angular dependence of the resonance parameters in the basal plane.

To investigate the magnetic structure of copper metaborate $\mathrm{CuB}_{2} \mathrm{O}_{4}$ in various regions of the phase diagram, neutron diffraction studies were done on a single crystal grown with the boron isotope ${ }^{11} \mathrm{~B}$ to reduce the neutron absorption. $^{10,13,15}$

The intensities of some of the Bragg peaks, especially those of the forbidden reflections (110) or (002), increase with decreasing temperature in the region $10-20 \mathrm{~K}$, indicating that an antiferromagnetic structure is realized in $\mathrm{CuB}_{2} \mathrm{O}_{4}$ in this temperature interval. In reciprocal lattice space such a structure is described by a propagation vector $\mathbf{q}=0$, so that the magnetic and crystal-chemical cells coincide. In this state the magnetic moments of the $\mathrm{Cu}(b)$ and $\mathrm{Cu}(d)$ ions are ordered differently: at $T=12 \mathrm{~K}$ the magnetic moments of the ions equal about $1 \mu_{B}$ per atom for $\mathrm{Cu}(b)$ and about $0.25 \mu_{B}$ for $\mathrm{Cu}(d)$. A detailed description of the magnetic structure of $\mathrm{CuB}_{2} \mathrm{O}_{4}$ in state 2 will be given below.

In the temperature region below $T_{I}$ magnetic satellites appear near the magnetic peaks on the neutronogram of $\mathrm{CuB}_{2} \mathrm{O}_{4}$; these satellites are located at symmetric positions with respect to the reciprocal lattice points of the commensurate phase (Fig. 9). Here the magnetic structure of copper metaborate becomes incommensurate along the tetragonal axis of the crystal and is described by a spin-density wave with phase modulation. ${ }^{10}$ The period of the spin modulation increases continuously from $q \approx 0$ near $10 \mathrm{~K}$ to $q$ $=(0,0,0.15)$ at a temperature of $1.8 \mathrm{~K}$ (Fig. 10). At this temperature the modulation of the spin structure is characterized by a period $c / 0.15 \approx 40 \AA$. The temperature dependence of the magnitude of the wave vector of the incommensurate phase of the spin structure obeys a power law:

$$
q(T)=A\left(T_{I}-T\right)^{0.5} .
$$

Relation (1) gives a good description of the temperature dependence of the wave vector of the incommensurate phase throughout the investigated temperature range (the solid curve in Fig. 10).

In a fitting of the magnetic structure at $T=2 \mathrm{~K}$ the best agreement is obtained for a simple helix with a magnetic moment $0.7 \mu_{B}$ for $\mathrm{Cu}(d)$.

A strong diffuse scattering superimposed on the Bragg peaks for neutron scattering vector $Q_{0}$ along the crystallographic direction [001]. The intensity of the diffuse scatter-

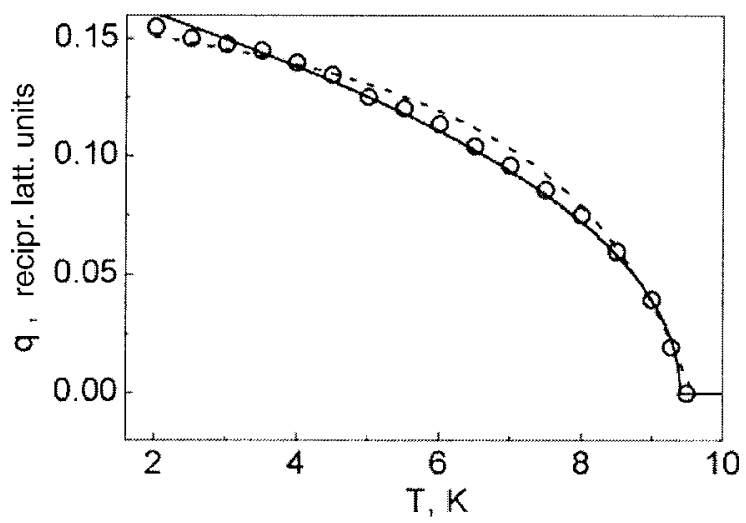

FIG. 10. Temperature dependence of the wave vector $q$ of the incommensurate structure. The solid curve was constructed from the power law (1), and the dashed curve is the result of modeling by the method of the phenomenological thermodynamic potential.

ing increases as the temperature is increased from $1.8 \mathrm{~K}$ and reaches a maximum value near the temperature $T_{I}$ (Ref. 10). The diffuse scattering is observed even at the lowest temperature reached in the experiment. This behavior differs markedly from the usual behavior of the spin subsystem of three-dimensional magnets with localized spins, for which critical fluctuations are limited to a small temperature region near the phase transition.

In addition to the first-order magnetic satellites corresponding to the wave vector $\mathbf{q}$ of the helix, higher-order satellites, the position of which corresponds to a wave vector $3 \mathbf{q}$, appear on the magnetic neutronogram near the temperature $T_{I}$ of the phase transition from the incommensurate to the commensurate structure (Fig. 11). ${ }^{10}$

The strong diffuse scattering of neutrons and the appearance of higher harmonics of the magnetic satellites near the temperature $T_{I}$ attest to the fact that the modulated structure is a lattice of magnetic solitons. Unlike a simple helix, such a magnetic structure can be represented in the form a helix with a nonconstant spatial rate of change of the phase of the modulation. If one moves along the $\mathbf{z}$ axis along the helix, there will be regions of slow variation of the phase, which can be attributed to domains, and regions of rapid variation-domain walls. This character of the modulation of the magnetic structure is due to magnetic anisotropy in the tetragonal plane of the crystal.

The neutron results presented above were obtained in the absence of an applied magnetic field. However, on the curves

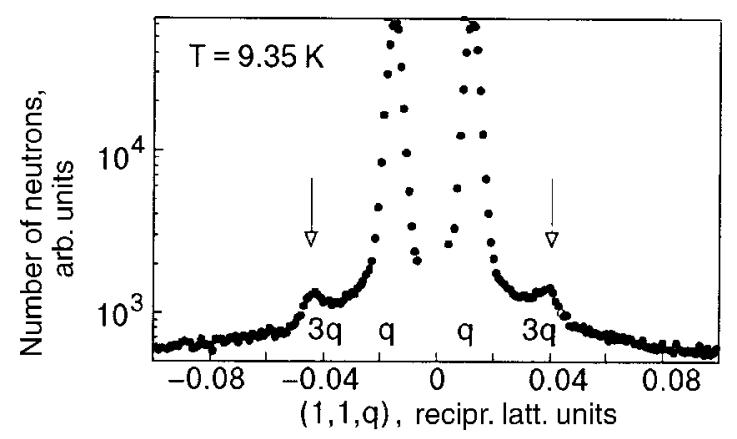

FIG. 11. Principal magnetic satellites $(1,1, q)$ and $(1,1,-q)$ and their higher harmonics at $T=9.35 \mathrm{~K}$. 


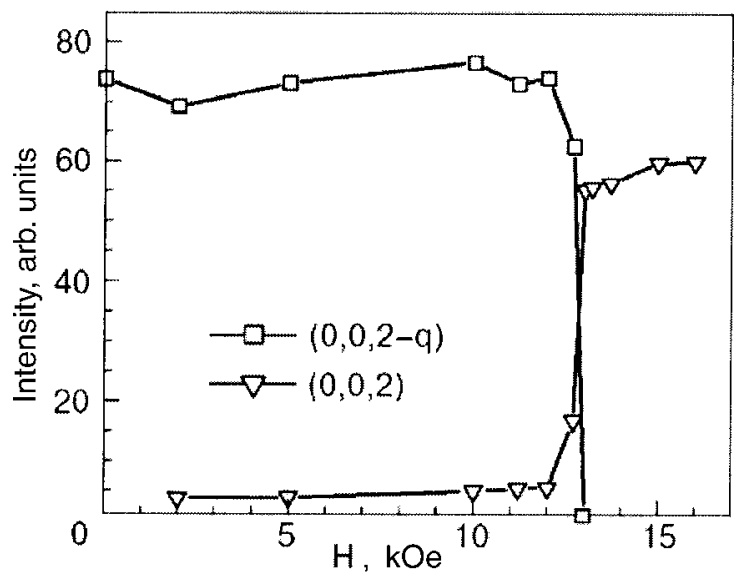

FIG. 12. Field dependence of the intensity of the reflections $(0,0,2)$ and $(0,0,2-q)$ in copper metaborate at $T=4.2 \mathrm{~K}$.

of the field dependence of the magnetization measured in a temperature interval $10-20 \mathrm{~K}$ in a magnetic field applied in the tetragonal plane of the crystal (Fig. 3), one observes a kink, the critical field of which increases with increasing temperature. Thus in this temperature interval in low fields there exists a transition from state 2 to a state 3 (see Fig. 7) in which there is no spontaneous magnetic moment. We assume that in this state the magnetic structure will also be modulated, but the modulation vector is considerably smaller than the resolving power of neutronography, so that it is impossible to distinguish between states 2 and 3 by neutron scattering.

Neutron-scattering studies ${ }^{15}$ done at $T=4.2 \mathrm{~K}$ in the presence of a magnetic field of up to $15 \mathrm{kOe}$ applied in the tetragonal plane confirmed that a transition from an incommensurate to a commensurate phase occurs at a field of around 13 kOe. It is seen in Fig. 12 that in the transition region there is a certain interval of fields in which on the neutronogram the central peak $(0,0,2)$, characteristic for the high-field commensurate phase, coexists with a magnetic satellite $(0,0,2-q)$, which belongs to the incommensurate state. Thus the transition in field from the incommensurate phase 1 to the commensurate phase 2 , in contrast to the analogous transition in temperature in the absence of magnetic field, is a first-order transition. This conclusion is confirmed by the field dependence of the wave vector of the helix, which decreases in a jump to zero when the magnetic field reaches the critical value $13 \mathrm{kOe}$. It should also be noted that in the presence of a magnetic field of $10 \mathrm{kOe}$ the higher harmonics of the magnetic satellites are observed throughout the temperature region in which the incommensurate state exists and not only near the phase transition temperature, as in the experiment with no magnetic field.

Neutron diffraction studies of the magnetic structure of $\mathrm{CuB}_{2} \mathrm{O}_{4}$ have also been done in the low-temperature region, ${ }^{13}$ where anomalies of the $\mu \mathrm{SR}$ and heat capacity are observed. It was found that the magnetic satellites are observed down to temperatures of $\sim 0.2 \mathrm{~K}$, i.e., the magnetic structure remains incommensurate, and no anomalies of the temperature dependence of the wave vector of the structure are observed in this region. However, the temperature dependence of the intensity of the magnetic satellites $(3,3,0-q)$

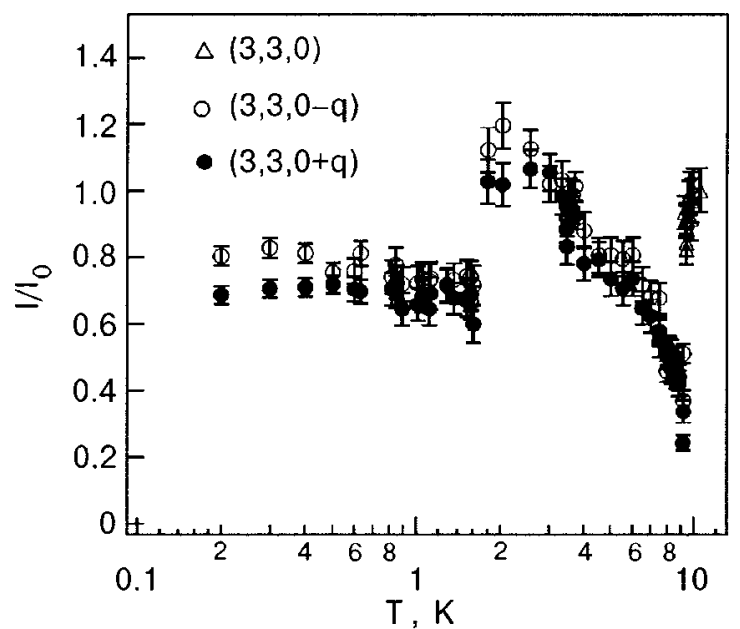

FIG. 13. Temperature dependence of the intensity of the magnetic reflections in copper metaborate.

and $(3,3,0+q)$ at a temperature of $1.8 \mathrm{~K}$ exhibits a downward jump of approximately $40 \%$ (Fig. 13). This indicates that the magnetic structure at $1.8 \mathrm{~K}$ undergoes a transformation, the nature of which is not yet understood.

\section{DISCUSSION OF THE RESULTS}

The set of experimental data presented above indicates that rearrangements of the spin system of copper metaborate occur at temperatures of 20,10 , and $2 \mathrm{~K}$. While the data are insufficient for analysis of the last of these phase transitions, we can draw the following conclusions about the first two.

In the commensurate phase 2 the magnetic structure is that of a weak ferromagnet. The propagation vector $\mathbf{q}=0$ corresponds to a coincidence of the magnetic and crystalchemical cells. The point group of the crystal is $\overline{4} 2 m$, which contains eight symmetry elements: ${ }^{16} 1, \overline{4}_{3}^{1}, 4_{3}^{2}, \overline{4}_{3}^{3}, 4_{1}^{2}, 4_{2}^{2}$, $m_{4}$, and $m_{5}$. At $\mathbf{q}=0$ this group has five irreducible representations. Four of them, $\Gamma_{1}, \Gamma_{2}, \Gamma_{3}$, and $\Gamma_{4}$, are onedimensional, and one of them, denoted $\Gamma_{5}$, is twodimensional. The decomposition of the representation for copper metaborate gives $\Gamma_{4 b}=\Gamma_{1}+\Gamma_{2}+2 \Gamma_{5}$, and $\Gamma_{8 d}=\Gamma_{1}$ $+2 \Gamma_{2}+2 \Gamma_{3}+\Gamma_{4}+3 \Gamma_{5}$. The magnetic modes $S_{b 1 z}+S_{b 2 z}$ and $S_{b 1 z}-S_{b 2 z}$, which correspond, respectively, to the representations $\Gamma_{2}$ and $\Gamma_{1}$ of the $4 b$ position, permit one to describe the ferromagnetic or antiferromagnetic ordering along the tetragonal axis c. The modes associated with the representation $\Gamma_{5}$, viz., $\left(S_{b 1 x}+S_{b 2 x},-S_{b 1 y}-S_{b 2 y}\right)$ and $\left(S_{b 1 y}-S_{b 2 y}, S_{b 1 x}-S_{b 2 x}\right)$, describe a noncollinear magnetic structure in the tetragonal plane. For the $8 d$ positions the magnetic modes are:

$$
\begin{array}{ll}
\Gamma_{1}: & S_{d 1 x}+S_{d 2 y}-S_{d 3 x}-S_{d 4 y} ; \\
\Gamma_{2}: & S_{d 1 y}-S_{d 2 x}-S_{d 3 y}+S_{d 4 x} \text { and } \\
& S_{d 1 z}+S_{d 2 z}+S_{d 3 z}+S_{d 4 z} ; \\
\Gamma_{3}: & S_{d 1 y}+S_{d 2 x}-S_{d 3 y}-S_{d 4 x} \text { and } \\
& S_{d 1 z}-S_{d 2 z}+S_{d 3 z}-S_{d 4 z} ;
\end{array}
$$




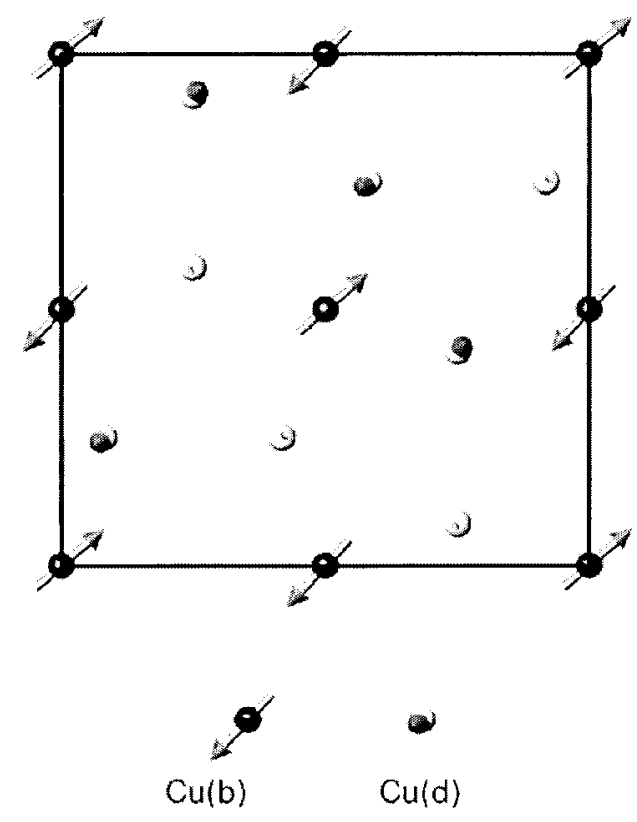

FIG. 14. Antiferromagnetic structure of copper metaborate in the commensurate state: $\mathrm{Cu}(b)$ and $\mathrm{Cu}(d)$ are represented by the dark and light symbols, respectively.

$$
\begin{aligned}
& \Gamma_{4}: \quad S_{d 1 x}-S_{d 2 x}-S_{d 3 y}+S_{d 4 y} ; \\
& \Gamma_{5}: \quad\left(S_{d 1 x}+S_{d 3 x},-S_{d 2 y}-S_{d 4 y}\right), \\
& \left(S_{d 2 x}+S_{d 4 x},-S_{d 1 y}-S_{d 3 y}\right) \text { and } \\
& \left(S_{d 2 z}-S_{d 4 z}, S_{d 1 z}-S_{d 3 z}\right) .
\end{aligned}
$$

Analysis of the neutron diffraction pattern, ${ }^{10}$ including 25 purely magnetic peaks, showed that the spins of both $\mathrm{Cu}(b)$ and $\mathrm{Cu}(d)$ of copper metaborate form a noncollinear magnetic structure (Fig. 14). In the spin subsystem of $\mathrm{Cu}(b)$ the antiferromagnetic vector in the tetragonal plane is dominant, and the vectors orthogonal to it-the ferromagnetic vector in the tetragonal plane and the antiferromagnetic vector along the $\mathbf{c}$ axis-are relatively small. In the spin subsystem of $\mathrm{Cu}(d)$ the antiferromagnetic vector along the $\mathbf{c}$ axis is dominant, and the orthogonal antiferromagnetic vector in the tetragonal plane is relatively small. The ferromagnetic vector in the plane is not detected within the experimental error limits. The magnetic moment of $\mathrm{Cu}(d)$ in the commensurate phase is much smaller than the magnetic moment of $\mathrm{Cu}(b)$. All of the vectors listed can be matched up with magnetic modes obtained from a symmetry analysis. It should be noted once again that, in spite of the fact that the magnetic structure presented is based on analysis of the neutron diffraction data obtained in the temperature range 10-20 $\mathrm{K}$ in the absence of an applied magnetic field, it corresponds to the state 2 induced by a small magnetic field on the phase diagram of Fig. 7. This conclusion is confirmed by the data of Ref. 13, in which a refinement of the magnetic structure was done at $T=12 \mathrm{~K}$ in a magnetic field of 400 Oe applied in the tetragonal plane. In accordance with the phase diagram, this field is sufficient to induce a transition of the crystal to state 2 at a temperature of $12 \mathrm{~K}$.
In the incommensurate phase 1 at $T<10 \mathrm{~K}$ the magnetic structure is ordered in the form of a helix. For theoretical analysis of the magnetic properties of copper metaborate by the method of the phenomenological thermodynamic potential it is important that there is no center of inversion $\overline{1}$ among its symmetry elements. It enters only in combination with a $90^{\circ}$ rotation about the c axis: $\overline{4}_{3}^{1}$ and $\overline{4}_{3}^{3}$. Therefore, it is permissible for the thermodynamic potential to contain an invariant of the Lifshitz type bilinear in the two-component order parameter and its spatial derivative.

In Ref. 17 such properties of a crystal as the temperature dependence of the wave vector of the modulation of the magnetic structure and heat capacity were analyzed in the framework of a phenomenological approach. The thermodynamic potential was written in the form of a functional of two two-component order parameters, $\left(\eta_{A 1}, \eta_{A 2}\right)$ and $\left(\eta_{B 1}, \eta_{B 2}\right)$ :

$$
\begin{aligned}
\Phi= & \int\left\{\frac{\alpha_{A}}{2} \eta_{A}^{2}+\frac{\beta_{A}}{4} \eta_{A}^{4}+\frac{\gamma_{A}}{4} \eta_{A}^{4} \cos \left(4 \varphi_{A}\right)\right. \\
& +\frac{\delta_{A}}{2}\left[\left(\nabla \eta_{A}\right)^{2}+\eta_{A}^{2}\left(\nabla \varphi_{A}\right)^{2}\right]-\sigma_{A} \eta_{A}^{2} \varphi_{A}^{\prime}+\frac{\alpha_{B}}{2} \eta_{B}^{2} \\
& +\frac{\beta_{B}}{4} \eta_{B}^{4}+\frac{\gamma_{B}}{4} \eta_{B}^{4} \cos \left(4 \varphi_{B}\right)+\frac{\delta_{B}}{2}\left[\left(\nabla \eta_{B}\right)^{2}\right. \\
& \left.\left.+\eta_{B}^{2}\left(\nabla \varphi_{B}\right)^{2}\right]-\sigma_{B} \eta_{B}^{2} \varphi_{B}^{\prime}+\kappa \eta_{A} \eta_{B} \cos \left(\varphi_{A}-\varphi_{B}\right)\right\} d V,
\end{aligned}
$$

where

$$
\begin{aligned}
& \alpha_{A}=\alpha_{A 0}\left(T-T_{A}\right), \quad \alpha_{B}=\alpha_{B 0}\left(T-T_{B}\right), \\
& \eta_{A}^{2}=\eta_{A 1}^{2}+\eta_{A 2}^{2}, \quad \varphi_{A}=\arctan \left(\eta_{A 2} / \eta_{A 1}\right), \\
& \eta_{B}^{2}=\eta_{B 1}^{2}+\eta_{B 2}^{2}, \quad \varphi_{B}=\arctan \left(\eta_{B 2} / \eta_{B 1}\right),
\end{aligned}
$$

$\alpha_{A 0}>0, \alpha_{B 0}>0, \beta_{A}>0, \beta_{B}>0, \delta_{A}>0, \delta_{B}>0, \nabla$ is the del operator, and $f^{\prime} \equiv d f / d z$. Both parameters transform according to the representation $\Gamma_{5}$, and they differ by a linear combination of magnetic modes transforming according to this representation. The parameter $\eta_{A}$ is associated with the transition at $20 \mathrm{~K}$ and, according to the magnetic and neutrondiffraction data given above, consists primarily of the modes $\left(S_{b 1 x}+S_{b 2 x},-S_{b 1 y}-S_{b 2 y}\right)$ and $\left(S_{b 1 y}-S_{b 2 y}, S_{b 1 x}-S_{b 2 x}\right)$. The description of the helical magnetic structure below $10 \mathrm{~K}$ is achieved by including in the thermodynamic potential a Lifshitz invariant constructed from the two-component parameter $\eta_{B}$. The presence in (2) of other order parameters is not decisive for the description of the evolution of the states in copper metaborate as the temperature is lowered from the paramagnetic phase above $20 \mathrm{~K}$ to the incommensurate phase below $10 \mathrm{~K}$.

The analysis is done in the approximation of a constant modulus of the order parameter, which is suitable for the case when the Lifshitz invariants and the invariants of the anisotropy and the interaction between subsystems are relatively small in comparison with the remaining invariants. 


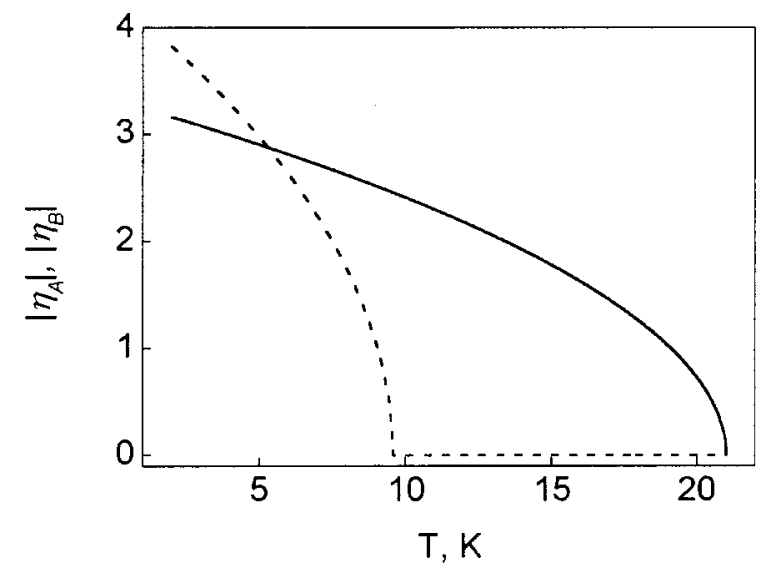

FIG. 15. Modeled temperature dependence of the moduli of the order parameters: $\left|\eta_{A}\right|$ (solid curve) and $\left|\eta_{B}\right|$ (dashed curve).

The resulting relations can be used to model the temperature dependence of $\eta_{A}, \eta_{B}$ and the wave number $q=2 \pi / \lambda$ (the dashed curve in Fig. 10), and, with the aid of the standard relation $C_{p}=-T \partial^{2} \Phi / \partial T^{2}$, the temperature dependence of the specific heat $c_{p}=C_{p} / V$ of the crystal for the following parameters of the thermodynamic potential:

$$
\begin{aligned}
& \alpha_{A}=1.6(T-20), \quad \beta_{A}=2.7, \quad \gamma_{A}=0, \\
& \delta_{A}=0.16, \sigma_{A}=0, \kappa=0.01, \\
& \alpha_{B}=4(T-9.6), \quad \beta_{B}=2, \gamma_{B}=0.01, \\
& \delta_{B}=0.16, \sigma_{B}=0.1 .
\end{aligned}
$$

The values of the parameters are given in kelvins. As we see from Fig. 15, in the temperature region 10-20 K the order parameter $\eta_{B}$ is small compared to $\eta_{A}$ and it increases rapidly with decreasing temperature below $10 \mathrm{~K}$. The analogous growth of the wave vector $q \equiv 2 \pi / \lambda$ in Fig. 10 agrees with the experimentally observed behavior (Figs. 9 and 10) but differs qualitatively in that $q$ is nonzero already at temperatures below $20 \mathrm{~K}$ : in the temperature region where the invariants of the anisotropy are small compared with the Lifshitz invariants, it it equal to

$$
q \approx \sigma / \delta, \text { where } \delta=\delta_{A} \eta_{A}^{2}+\delta_{B} \eta_{B}^{2}, \quad \sigma=\sigma_{A} \eta_{A}^{2}+\sigma_{B} \eta_{B}^{2},
$$

and for $\sigma_{A}=0$ it varies basically in the same way as $\eta_{B}$.

In calculating the heat capacity we took into account not only the jump (described by Landau theory) at the transition and the Ginzburg estimate for the contribution of thermal fluctuations of the order parameter but also the contributions of acoustic phonons and anomalies of the Schottky type. At a temperature of $20 \mathrm{~K}$ the fluctuation contribution inherent to a second-order phase transition, with a maximum at the transition point is dominant. The experimental curves of the temperature dependence of the heat capacity of a $\mathrm{CuB}_{2} \mathrm{O}_{4}$ single crystal (Fig. 4) have a step-like feature at $9.6 \mathrm{~K}$. It is due to the rapid growth of the order parameter in the second spin subsystem, which is nonzero already at temperatures below $20 \mathrm{~K}$ because of the bilinear interaction with the first subsystem. The field induced by this interaction suppresses the thermal fluctuations in the second subsystem. For this reason the latter were not taken into account in the calculation. We note that the step at $9.6 \mathrm{~K}$ is observed against the background of a broad maximum that increases with decreasing temperature; this maximum can be attributed to a Schottky-type anomaly not described in the phenomenological approach. The deviation of the experimental from the calculated dependence of the heat capacity as the temperature is lowered below $3.5 \mathrm{~K}$ is due to the approach to the transition near $2 \mathrm{~K}$.

Thus a comprehensive set of experimental and theoretical studies has shown that in the temperature region 10-20 K the $\mathrm{CuB}_{2} \mathrm{O}_{4}$ crystal has a long-period modulated magnetic structure with a small propagation vector along the tetragonal axis of the crystal. Under the influence of a weak (less than $1 \mathrm{kOe}$ ) magnetic field applied in the tetragonal plane, a transition occurs to a commensurate noncollinear magnetic structure that is formed by two subsystems of copper ions and is characterized by the presence of a spontaneous magnetic moment. Below $10 \mathrm{~K}$ the magnetic structure of this compound is an incommensurate soliton lattice with a propagation vector directed along the tetragonal axis of the crystal.

This study was supported by the Russian Foundation for Basic Research, Grant 01-02-17270.

*E-mail: pank@iph.krasn.ru

${ }^{1}$ G. Petrakovskii, K. Sablina, A. Pankrats, A. Vorotinov, A. Furrer, B. Roessli, and P. Fischer, J. Magn. Magn. Mater. 140-144, 1991 (1995).

${ }^{2}$ G. A. Petrakovski1, K. A. Sablina, A. M. Vorotynov, A. I. Kruglik, A. G. Klimenko, A. D. Balaev, and S. S. Aplesnin, Zh. Éksp. Teor. Fiz. 98, 1382 (1990) [Sov. Phys. JETP 71, 772 (1990)].

${ }^{3}$ G. A. Petrakovskiŭ, Izv. Vyssh. Uchebn. Zaved. Fiz., No. 1, 91 (1998).

${ }^{4}$ A. M. Vorotynov, A. I. Pankrats, G. A. Petrakovskiǔ, K. A. Sablina, V. Pashkovich, and G. Shimchak, Zh. Éksp. Teor. Fiz. 113, 1866 (1998) [JETP 86, 1020 (1998)].

${ }^{5}$ G. A Petrakovskii, K. S. Aleksandrov, L. N. Bezmaternikh, S. S. Aplesnin, B. Roessli, F. Semadeni, A. Amato, C. Baines, J. Bartolome, and M. Evangelisti, Phys. Rev. B 63, 184425 (2001).

${ }^{6}$ G. A. Petrakovskiı̌, K. A. Sablina, A. I. Pankrats, D. A. Velikanov, A. D. Balaev, O. A. Bayukov, V. I. Tugarinov, A. M. Vorotynov, A. D. Vasil'ev, G. V. Romanenko, and Yu. G. Shvedenkov, Fiz. Tverd. Tela (St. Petersburg) 44, 1280 (2002) [Phys. Solid State 44, 1339 (2002)].

${ }^{7}$ Yu. A. Izyumov, Neutron Diffraction on Long-Period Structures [in Russian], Energoatomizdat, Moscow (1987).

${ }^{8}$ I. E. Dzyaloshinskiǔ, Zh. Éksp. Teor. Fiz. 47, 992 (1964) [Sov. Phys. JETP 20, 665 (1965)].

${ }^{9}$ G. A. Petrakovskiĭ, K. A. Sablina, D. A. Velikanov, A. M. Vorotynov, N. V. Volkov, and A. F. Bovina, Kristallografiya 45, 926 (2000) [Crystallogr. Rep. 45, 853 (2000)].

${ }^{10}$ B. Roessli, J. Shefer, G. Petrakovskii, B. Ouladdiaf, M. Boehm, U. Staub, A. Vorotinov, and L. Bezmaternikh, Phys. Rev. Lett. 86, 1885 (2001).

${ }^{11}$ G. Petrakovskii, D. Velikanov, A. Vorotinov, A. Balaev, K. Sablina, A. Amato, B. Roessli, J. Schefer, and U. Staub, J. Magn. Magn. Mater. 205, 105 (1999).

${ }^{12}$ G. A. Petrakovski1̌, A. D. Balaev, and A. M. Vorotynov, Fiz. Tverd. Tela (St. Petersburg) 42, 313 (2000) [Phys. Solid State 42, 321 (2000)].

${ }^{13}$ M. Boehm, B. Roessli, J. Shefer, B. Ouladdiaf, A. Amato, C. Baines, U. Staub, and G. Petrakovskii, Physica B (2002) (in press).

${ }^{14}$ A. I. Pankrats, G. A. Petrakovskiǐ, and N. V. Volkov, Fiz. Tverd. Tela (St. Petersburg) 42, 93 (2000) [Phys. Solid State 42, 96 (2000)].

${ }^{15}$ J. Shefer, M. Boehm, B. Roessli, G. Petrakovskii, B. Ouladdiaf, and U. Staub, Appl. Phys. A: Solids Surf. (2002) (in press).

${ }^{16} \mathrm{O}$. V. Kovalev, Irreducible and Induced Representations and Corepresentions of Fedorov Groups [in Russian], Nauka, Moscow (1986).

${ }^{17}$ G. A. Petrakovskii, M. A. Popov, B. Roessli, and B. Ouladdiaf, Zh. Éksp. Teor. Fiz. 120, 926 (2001) [JETP 93, 809 (2001)]

Translated by Steve Torstveit 